\title{
Resposta cardiorrespiratória na asma induzida pelo exercício máximo com incrementos progressivos*
}

\author{
Ruy Amazonas Lamar Filho ${ }^{1}$, Antonio Augusto SoARes da FonseCA², \\ Maria Alice Melo NeVES ${ }^{3}$, LAÉrCio Moreira VAlenÇA ${ }^{4}$
}

Objetivo: Verificar a ocorrência de broncoconstrição induzida por exercício e verificar a resposta cardiorrespiratória durante o exercício máximo em pacientes asmáticos. Pacientes e métodos: Quatorze asmáticos ( $\mathrm{VEF}_{1}$ basal de 86,3\%), conforme os critérios da American Thoracic Society, foram submetidos a teste de exercício máximo. Foram realizadas curvas fluxo-volume antes, sete e 15 minutos após esforço progressivo máximo. Seis indivíduos (43\%) apresentaram queda do $\mathrm{VEF}_{1}$ igual ou maior que $15 \%$ após esforço máximo (grupo I). Os demais constituíram o grupo II. No grupo I, observou-se redução no $\mathrm{VEF}_{1}$ de $40,9 \%$ e $26,7 \%$ sete e 15 min após o exercício, enquanto no grupo II ocorreu diminuição de $2,6 \%$ e aumento de $1,2 \%$. No pico do exercício, os dois grupos atingiram FCmax acima de $91 \%$ do valor de referência; a carga máxima foi de $82,7 \%$ e $62,5 \%$, o 을 $_{2}$ max (mL/ $\mathrm{kg} / \mathrm{min}$ ) de $93,5 \%$ e $58,9 \%$ e a VEmax de $91,5 \%$ e $63,8 \%$, respectivamente, nos grupos I e II. Essas diferenças foram estatisticamente significativas. A correlação da queda percentual do $\mathrm{VEF}_{1}$ (7min pósexercício) com a VEmax (\%predito) mostrou um coeficiente $\mathrm{r}=0,8989$ para o grupo I e um $\mathrm{r}=$ 0,3629 para o grupo II. Não se observou correlação estatisticamente significativa entre o delta $\mathrm{VEF}_{1} e$ $\mathrm{O}$ v $\mathrm{O}_{2} \max$ (\% predito). Conclusão: Nos pacientes com asma induzida pelo exercício, a ocorrência de broncoconstrição correlacionou-se com o nivel de ventilação máxima, mas não com a aptidão física (v을 máximo). (J Pneumol 2001;27(3):137-142)

\section{Cardiorespiratory response to incremental progressive maximal exercise in asthmatic patients}

Objective: To determine the occurrence of exercise-induced bronchoconstriction and analyze cardiorespiratory response during maximal exercise in asthmatic patients. Patients and methods: Fourteen subjects with asthma ( $F E V_{1}$ of $86.3 \%$ predicted), as defined by the American Thoracic Society criteria were submitted to a maximal exercise test. Volume-flow curves were performed prior to progressive maximal exercise and seven and 15 minutes after it. Six patients (43\%) showed a decline in $\mathrm{FEV}_{1}$ equal or greater than $15 \%$ after exercise (group I). The remaining

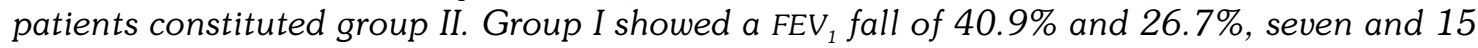
minutes after exercise, respectively. In group II, there was a decrease of $2.6 \%$, after 7 min and an increase of $1.2 \%$ after $15 \mathrm{~min}$. At peak exercise, both groups reached heart rate above $91 \%$ of reference values; peak work was $82.7 \%$ and $62.5 \%, \mathrm{VO}_{2} \max (\mathrm{mL} / \mathrm{kg} / \mathrm{min}) 93.5 \%$ and $58,9 \%$ and VEmax $91.5 \%$ and $63.8 \%$, respectively, in groups I and II. These differences were statistically significant. The correlation between the FEV fall rate $(7 \mathrm{~min}$ post-exercise) and VEmax (\% predicted) has showed a coefficient $r=0.8989$ in group I and $r=0.3629$ in group II. There was no correlation between delta $\mathrm{VEF}_{1}$ and $\mathrm{VO}_{2} \max$ (\% predicted) in both groups. These findings showed that, in exercise-induced asthma patients, the occurrence of bronchoconstriction correlated with the level of maximal ventilation, but not with physical fitness $\left(\dot{\mathrm{V}}_{2}\right.$ max).

* Trabalho realizado na Clínica Pneumológica do Hospital das Forças Armadas, Brasília, DF.

1. Médico Assistente.

2. Ex-Médico Residente.

3. Fisioterapeuta.

4. Chefe da Clínica.
Endereço para correspondência - Laércio M. Valença, Clínica Pneumológica, Hospital das Forças Armadas - 70630-900 - Brasília, DF. Tel. (61) 362-4218; fax (61) 346-9322; E-mail: valenca@essencial. com.br

Recebido para publicação em 20/6/00. Aprovado, após revisão, em 14/7/00. 
Descritores - Asma induzida pelo exercício. Broncoconstrição. Volume de ventilação pulmonar. Teste de função respiratória.

Key words - Exercise induced asthma. Bronchoconstriction. Tidal volume. Respiratory function tests.

\section{INTRODUÇÃO}

Asma induzida pelo exercício (AIE) ou broncoconstrição induzida pelo exercício (BIE) são termos sinônimos, usados para descrever o aumento transitório na resistência das vias aéreas após um exercício vigoroso, o que pode ocorrer na maioria dos pacientes com asma ${ }^{(1)}$. É reconhecida como importante fator limitante da atividade física em jovens.

Os aspectos clínicos da AIE são habitualmente característicos. Pacientes sensiveis a esse estímulo podem tipicamente completar um exercício sem dificuldade. Quando param, contudo, desenvolvem por tempo limitado constrição torácica, chiado, dispnéia e tosse. A broncoconstrição atinge o seu pico entre cinco e dez minutos após o exercício e, a seguir, sobrevém uma remissão espontânea, que se completa entre 20 e 40 minutos. O exercício nunca causa estado de mal asmático e/ou episódios recorrentes de broncoespasmo ${ }^{(2,3)}$.

A corrida livre, o exercício em esteira e em cicloergômetro são as modalidades mais comumente empregadas para detectar a presença de BIE. Os protocolos usam geralmente um exercício de cinco a oito minutos, que deve ser bastante vigoroso para acelerar a freqüência cardíaca e o consumo de oxigênio a mais de $80 \%$ do valor máximo. Reiff et al. ${ }^{(4)}$ definiram AIE por queda maior de que $15 \%$ no $\mathrm{VEF}_{1}$ após um exercício padrão em esteira (6kph com inclinação de $15 \%$ durante seis minutos). Outros autores ${ }^{(5)}$, utilizando protocolo diferente, usaram o mesmo ponto de corte. Na literatura(6,7), no entanto, aceita-se redução de 10 a $20 \%$ do $\mathrm{VEF}_{1}$ ou pico do fluxo expiratório (PFE) como limite de resposta.

A broncoconstrição induzida pelo exercício (BIE) ocorre em 40 a $90 \%$ dos asmáticos, dependendo do tipo de exercício empregado e das condições em que foi realizado. Em proporção menor, a BIE ocorre também em indivíduos atópicos e em parentes de asmáticos ${ }^{(8,9)}$. A corrida livre é o tipo de exercício mais implicado na gênese da AIE, enquanto a natação $e$ a caminhada têm o menor potencial de provocação. $\mathrm{O}$ ciclismo e a canoagem situam-se entre esses extremos ${ }^{(10)}$. Estudos mais recentes, entretanto, mostraram que a intensidade da BIE é função do volume de ar ventilado e da diminuição da temperatura e umidade no ar inalado(5,11).

A despeito da extensa literatura sobre diversos aspectos da AIE, tem sido dada relativamente pouca atenção à
Siglas e abreviaturas utilizadas neste trabalho

AIE - Asma induzida pelo exercício

BIE - Broncoconstrição induzida pelo exercício

$\mathrm{VEF}_{1}$ - Volume expiratório forçado no primeiro segundo

CVF - Capacidade vital forçada

$\mathrm{FEF}_{25-75}$ - Fluxo expiratório forçado a 25-75\% da CVF

PFE - Pico do fluxo expiratório

FC - Freqüência cardíaca

FR - Freqüência respiratória

VC - Volume corrente

$\mathrm{FEO}_{2}$ - Fração de oxigênio no ar expirado misto

$\mathrm{FECO}_{2}$ - Fração de $\mathrm{CO}_{2}$ no ar expirado misto

FCmax - Frequência cardíaca máxima no pico do exercício

VEmax - Ventilação minuto máxima ou no pico do exercício

$\dot{\mathrm{v}} \mathrm{O}_{2} \mathrm{max}$ - Consumo de oxigênio máximo ou no pico do exercício

VCmax - Volume corrente máximo ou no pico do exercício

resposta cardiorrespiratória durante o exercício máximo em pacientes asmáticos. Assim, o presente trabalho tem como objetivo analisar essa resposta como resultado da aplicação de um teste cicloergométrico máximo com incrementos progressivos.

\section{MATERIAL E MÉTODOS}

Foram estudados 14 indivíduos portadores de asma, diagnosticada de acordo com critérios propostos pela American Thoracic Society ${ }^{(12)}$, com $\mathrm{VEF}_{1}$ médio de $86,3 \%$ (variação de $62 \%$ a $110 \%$ ) no teste pré-exercício, com idade média de 17 anos, sendo oito do sexo masculino, selecionados entre aqueles que estavam em acompanhamento ambulatorial por ser jovens, com asma de leve a moderada ${ }^{(13)}$ e sem história prévia de tabagismo e de práticas desportivas regulares. Dois terços dos pacientes faziam uso de drogas beta-2-agonistas, quando necessário, para o controle de sintomas asmáticos; os demais utilizavam regularmente corticosteróides inalatórios.

Todos se abstiveram do uso de broncodilatadores pelo menos durante as seis horas anteriores à realização dos testes de função pulmonar. Em um espirômetro a ar seco OHIO-842, os asmáticos realizaram curvas fluxo-volume antes, sete e 15 minutos após esforço progressivo máximo. Foram registrados o volume expiratório forçado no primeiro segundo $\left(\mathrm{VEF}_{1}\right)$, a capacidade vital forçada (CVF), o fluxo expiratório forçado a $25-75 \%$ da $\mathrm{CVF}\left(\mathrm{FEF}_{25-75}\right)$ e o pico do fluxo expiratório (PFE), sendo escolhidos os melhores valores.

O teste cicloergométrico consistiu em incrementos de 25 watts a cada dois minutos até levar os indivíduos à exaustão. Foram registrados continuamente em um polígrafo Hewlett-Packard (sistema 47606 A) a freqüência cardíaca (FC), a freqüência respiratória (FR), a ventilação minuto (VE), as frações de oxigênio e $\mathrm{CO}_{2}$ no ar expirado 
misto $\left(\mathrm{FEO}_{2}, \mathrm{FECO}_{2}\right)$ e calculado o valor do $\dot{\mathrm{vO}}_{2}$ e $\dot{\mathrm{v}} \mathrm{CO}_{2}$. Os detalhes desse protocolo foram descritos previamente ${ }^{(14)}$. Os exames foram realizados com os pacientes respirando ar ambiente, à temperatura média de $25^{\circ} \mathrm{C}$.

Os resultados dos testes espirométricos e de exercício foram examinados como percentagens dos valores preditos ${ }^{(15-17)}$. A variação dos testes espirométricos em relação ao valor pré-exercício foi calculada de forma igual à empregada no cálculo do $\mathrm{VEF}_{1}$ : delta $\mathrm{VEF}_{1}=\left(\mathrm{VEF}_{1}\right.$ pré-teste menor valor pós-teste)/VEF ${ }_{1}$ pré-teste x 100 .

Dos 14 indivíduos asmáticos, seis (43\%) apresentaram queda do $\mathrm{VEF}_{1}$ igual ou maior que $15 \%$ após o exercício, caracterizando a ocorrência de BIE. Esse grupo, constituído por três homens e três mulheres, foi denominado grupo I. Os demais pacientes constituíram o grupo II.

O teste $t$ de Student foi aplicado para comparar os parâmetros cardiorrespiratórios durante exercício máximo entre os grupos I e II. E a análise de regressão linear simples de Pearson foi usada para a correlação ( $\mathrm{r}$ ) entre o delta $\mathrm{VEF}_{1}$ sete minutos pós-exercício com a ventilação minuto (VE) e o consumo de oxigênio $\left(\mathrm{v}_{2}\right)$ atingidos no pico do exercício. O nível de significância estatística foi estabelecido para $\mathrm{p}<0,05$.

\section{TABELA 1}

Dados espirométricos (média e desvio padrão) do grupo I antes, sete e 15 minutos após o exercício

\begin{tabular}{lrrr}
\hline & Antes & Sete minutos & 15 minutos \\
\hline VEF $_{1}(\mathrm{~L})$ & $2,55 \pm 0,97$ & $1,57 \pm 0,97$ & $1,89 \pm 0,82$ \\
$\mathrm{VEF}_{1}(\%)$ & $89,5 \pm 18,3$ & $55,2 \pm 28,8$ & $68,0 \pm 44,9$ \\
$\mathrm{CVF}(\mathrm{L})$ & $3,60 \pm 1,11$ & $2,58 \pm 1,59$ & $3,01 \pm 1,10$ \\
$\mathrm{CVF}(\%)$ & $101,2 \pm 10,4$ & $71,8 \pm 40,1$ & $85,0 \pm 20,6$ \\
$\mathrm{VEF}_{1} / \mathrm{CVFx} 100$ & $70,6 \pm 12,4$ & $64,3 \pm 18,4$ & $62,0 \pm 14,9$ \\
$\mathrm{PFE}(\mathrm{L} / \mathrm{min})$ & $392,8 \pm 94,8$ & $230,5 \pm 124,3$ & $244,2 \pm 88,2$ \\
$\mathrm{PFE}(\%)$ & $89,3 \pm 18,3$ & $51,3 \pm 21,7$ & $56,5 \pm 21,0$ \\
$\mathrm{FEF}_{25-75}(\mathrm{~L} / \mathrm{S})$ & $3,05 \pm 1,27$ & $1,37 \pm 0,82$ & $1,96 \pm 1,08$ \\
$\mathrm{FEF}_{25-75}(\%)$ & $84,5 \pm 24,3$ & $38,5 \pm 22,6$ & $57,3 \pm 35,7$ \\
\hline
\end{tabular}

TABELA 2

Dados espirométricos (média e desvio padrão) do grupo II antes, sete e 15 minutos após o exercício

\begin{tabular}{lccc}
\hline & Antes & Sete minutos & $\mathbf{1 5}$ minutos \\
\hline $\mathrm{VEF}_{1}(\mathrm{~L})$ & $3,11 \pm 0,82$ & $3,04 \pm 0,83$ & $3,14 \pm 0,88$ \\
$\mathrm{VEF}_{1}(\%)$ & $83,9 \pm 16,7$ & $81,6 \pm 18,2$ & $84,7 \pm 19,4$ \\
$\mathrm{CVF}(\mathrm{L})$ & $4,08 \pm 0,93$ & $3,92 \pm 0,99$ & $4,05 \pm 0,91$ \\
$\mathrm{CVF}(\%)$ & $88,4 \pm 13,5$ & $84,6 \pm 15,2$ & $87,7 \pm 13,8$ \\
$\mathrm{VEF}_{1} / \mathrm{CVFx} 100$ & $75,9 \pm 7,18$ & $77,5 \pm 7,08$ & $77,3 \pm 9,09$ \\
$\mathrm{PFE}(\mathrm{L} / \mathrm{min})$ & $440,4 \pm 107,9$ & $472,5 \pm 191,5$ & $429,6 \pm 101,8$ \\
$\mathrm{PFE}(\%)$ & $83,6 \pm 14,1$ & $89,4 \pm 32,6$ & $82,5 \pm 15,0$ \\
$\mathrm{FEF}_{25-75}(\mathrm{~L} / \mathrm{S})$ & $4,07 \pm 1,70$ & $3,64 \pm 2,09$ & $4,24 \pm 1,94$ \\
$\mathrm{FEF}_{25-75}(\%)$ & $96,7 \pm 36,5$ & $85,1 \pm 46,7$ & $100,5 \pm 43,1$ \\
\hline
\end{tabular}

\section{RESULTADOS}

Os achados espirométricos nos grupos I e II antes, sete e 15 minutos após o exercício máximo, com incrementos progressivos, são mostrados nas Tabelas 1 e 2 . Antes do exercício, o grau de limitação do fluxo aéreo em ambos os grupos foi de intensidade discreta, com $\mathrm{VEF}_{1}$ de $89,5 \%$ e $83,9 \%$ em relação aos valores $\operatorname{previstos}^{(13)}$ e a relação $\mathrm{VEF}_{1} / \mathrm{CVF}$ de $70,6 \%$ e $75,9 \%$, respectivamente.

A redução do $\mathrm{VEF}_{1}$, PFE e $\mathrm{FEF}_{25-75}$ em relação aos valores pré-exercício foi, em média, de $46,9 \%$ aos sete minutos e de $34,0 \%$ aos 15 minutos no grupo I. No grupo II, a diminuição foi de $3,8 \%$ aos sete minutos e houve aumento de $1,3 \%$ aos 15 minutos. Sete minutos após o exercício, a queda mais importante entre os indicadores de fluxo foi do $\mathrm{FEF}_{25-75}(57,0 \%)$ e, a menos intensa, do $\mathrm{VEF}_{1}$ (40,9\%). A modificação percentual dos fluxos aéreos e da capacidade vital forçada em relação aos valores pré-exercício está mostrada no Gráfico 1.

Os parâmetros cardiorrespiratórios registrados no pico do exercício máximo progressivo (Tabela 3) mostraram que o grupo I atingiu FCmax de $96,7 \%$ do previsto ${ }^{(14,15)} e$ o grupo II, FCmax de 91,7\% ( $p=0,36$ ), o que indica ter existido empenho geral dos participantes em alcançar o esforço máximo. Ainda em relação aos citados valores preditos, a carga máxima foi de $82,7 \%$ e $62,5 \%$ ( $p=$ 0,0043), o $\dot{\mathrm{v}}_{2} \max (\mathrm{mL} / \mathrm{min})$, de $97,8 \%$ e $61,4 \%(\mathrm{p}=$ 0,0082), o $\dot{\mathrm{VO}}_{2} \max (\mathrm{mL} / \mathrm{kg} / \mathrm{min})$, de $93,5 \%$ e $58,9 \%$ (p $=0,0039)$, a VEmax, de 91,5\% e 63,8\% (p = 0,0476) e o VCmax, de $114,2 \%$ e $81,5 \%$ ( $p=0,0256$ ) nos grupos I e II, respectivamente. Essas diferenças foram estatisticamente significativas, indicando que os pacientes com AIE não só realizaram exercício mais intenso, como alcançaram ventilação minuto significativamente maior à custa do aumento do volume corrente, já que não houve diferença em relação à freqüência respiratória.

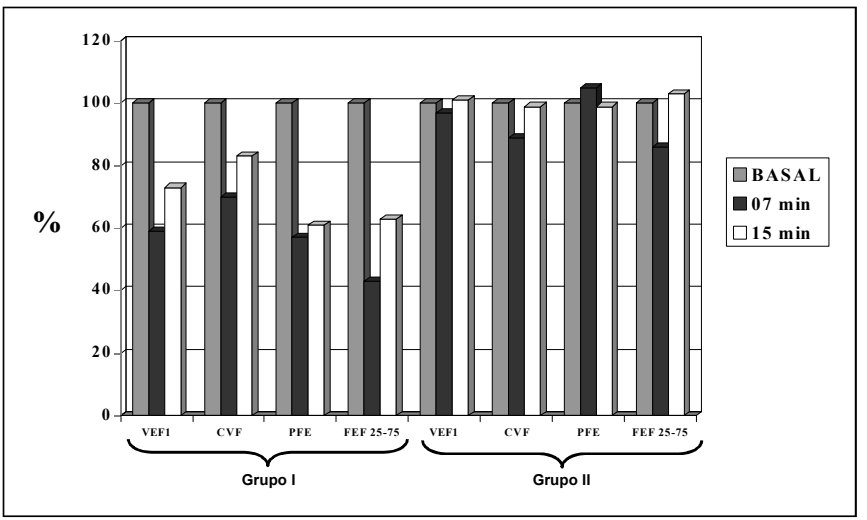

Gráfico 1 - Redução dos índices de fluxo e da CVF sete e 15 minutos após exercício máximo em comparação com os valores préexercício nos grupos I (com BIE) e II (sem BIE) 
TABELA 3

Dados antropométricos e parâmetros cardiorrespiratórios obtidos no pico do exercício progressivo máximo (média e desvio padrão)

\begin{tabular}{|c|c|c|c|}
\hline & Grupo I & Grupo II & Valor de $p$ \\
\hline Idade & $17,17 \pm 5,53$ & $17,88 \pm 4,09$ & \\
\hline Peso (kg) & $48,17 \pm 18,80$ & $61,13 \pm 11,23$ & \\
\hline Altura $(\mathrm{cm})$ & $159,17 \pm 14,19$ & $169,63 \pm 7,93$ & \\
\hline Carga max (W) & $175 \pm 52,44$ & $159 \pm 51,65$ & NS \\
\hline Carga max $\%$ predito & $82,7 \pm 9,58$ & $62,5 \pm 11,3$ & 0,0043 \\
\hline Freq. cardíaca max/min & $192 \pm 10,09$ & $182 \pm 24,66$ & NS \\
\hline Freq. cardíaca max \% pred & $96,7 \pm 5,39$ & $91,7 \pm 11,80$ & 0,3648 \\
\hline $\mathrm{VO}_{2} \max (\mathrm{mL} / \mathrm{min})$ & $2.074,3 \pm 879,46$ & $1.715,1 \pm 802,20$ & NS \\
\hline $\mathrm{VO}_{2} \max (\mathrm{mL} / \mathrm{min}) \%$ pred & $97,8 \pm 23,3$ & $61,4 \pm 19,8$ & 0,0082 \\
\hline $\mathrm{VO}_{2} \max (\mathrm{mL} / \mathrm{min} / \mathrm{kg})$ & $32,5 \pm 7,71$ & $24,2 \pm 10,55$ & NS \\
\hline $\mathrm{VO}_{2} \max (\mathrm{mL} / \mathrm{min} / \mathrm{kg}) \%$ pred & $93,5 \pm 15,7$ & $58,9 \pm 18,4$ & 0,0039 \\
\hline $\mathrm{VCO}_{2} \max (\mathrm{mL} / \mathrm{min})$ & $2.126 \pm 777,92$ & $1.891 \pm 926,99$ & NS \\
\hline VEmax (L/min) & $77,67 \pm 29,30$ & $70,75 \pm 34,76$ & NS \\
\hline VEmax (L/min) \% predito & $91,5 \pm 26,4$ & $63,7 \pm 20,8$ & 0,0476 \\
\hline Freq. respiratória max/min & $47,33 \pm 12,24(32-68)$ & $42,00 \pm 12,65$ & 0,4411 \\
\hline Volume corrente max & $2.001,67 \pm 935,59$ & $1.741,75 \pm 767,23$ & NS \\
\hline Volume corrente max $\%$ pred & $114,2 \pm 28,3$ & $81,5 \pm 19,4$ & 0,0256 \\
\hline
\end{tabular}

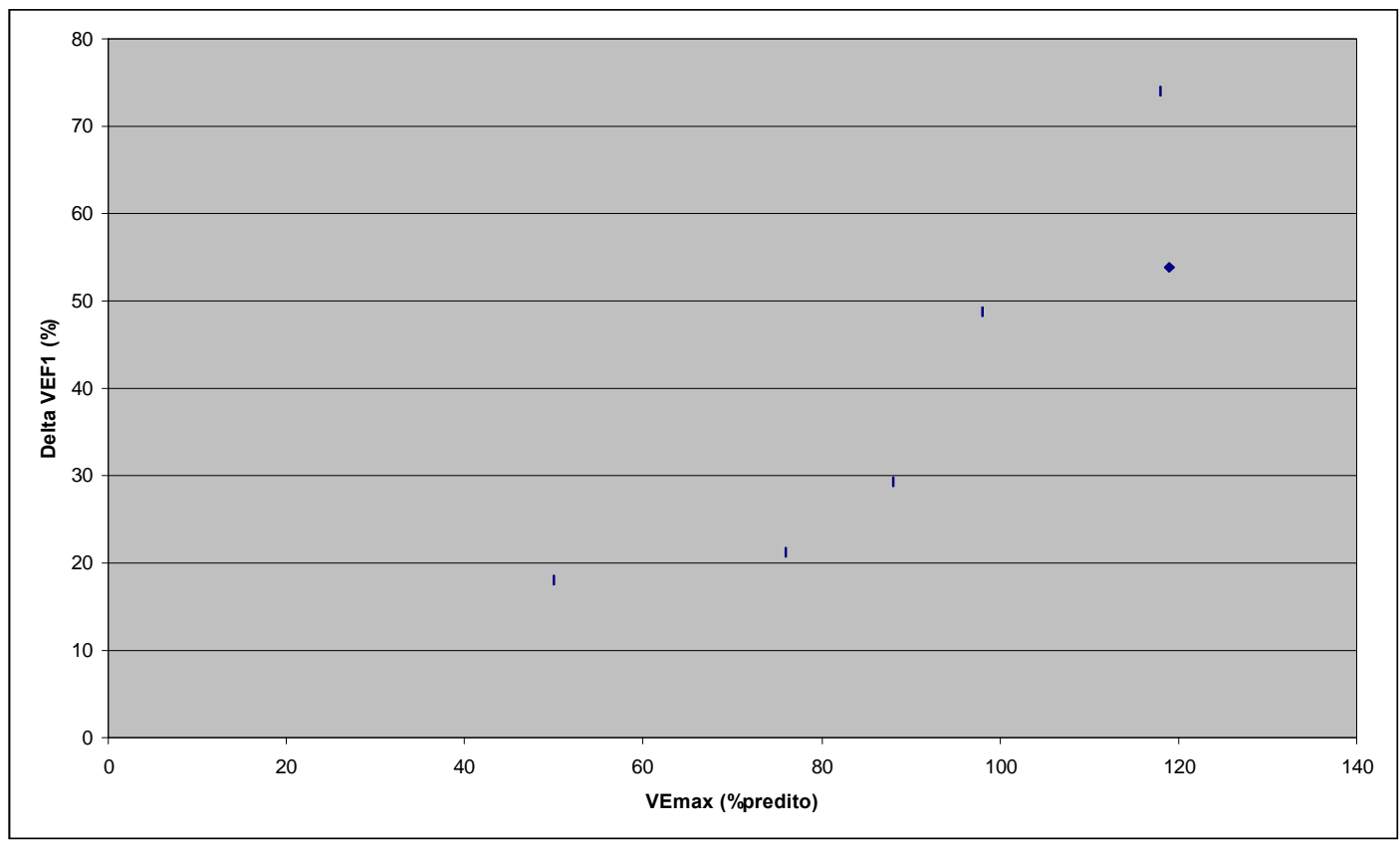

Gráfico 2 - Correlação do delta $V E F_{1}(\%)$, sete minutos pós-exercício, com a ventilação minuto (\% predito), no pico do exercício, nos pacientes com AIE $(r=0,8989)$

As correlações da queda percentual do $\mathrm{VEF}_{1}$ (sete min pós-exercício) com a VEmax (\% predito) mostraram coeficiente de 0,8989 para o grupo I $(\mathrm{p}=0,015)$ e de 0,3629 para o grupo II $(p=0,38)$, demonstrando, assim, que nos pacientes com BIE, quanto mais alta a ventilação minuto alcançada, maior o grau de obstrução ao fluxo aéreo, traduzido pela queda observada no $\operatorname{VEF}_{1}$ (Gráfico 2). Já o coeficiente de correlação entre o delta $\mathrm{VEF}_{1}$ e o $\mathrm{vO}_{2} \max$ foi de $r=0,3538(p=0,49)$ e $r=0,4047(p=0,32)$ nos grupos I e II, respectivamente. 


\section{DISCUSSÃO}

O presente estudo demonstrou a ocorrência de AIE em $43 \%$ dos asmáticos estudados (grupo I). A queda máxima do $\mathrm{VEF}_{1}$ pós-exercício nesse grupo foi de $40,9 \%$, proporcionalmente maior que a taxa de $22 \%$ descrita por Bierman et al. (9) e inferior aos valores obtidos por Reiff et al. ${ }^{(4)}$, cuja média foi de $46 \%$. Entre as medidas espirométricas, o $\mathrm{FEF}_{25-75}$ apresentou a maior queda percentual (57\%) sete minutos depois de findo o exercício. Outros autores encontraram, igualmente, diminuição maior do $\mathrm{FEF}_{25-75} \mathrm{em}$ relação ao $\mathrm{VEF}_{1}{ }^{(18,19)}$. Tal et al. ${ }^{(20)}$ estudaram sete asmáticos com BIE e seis sem BIE com teste de esforço máximo, com incrementos progressivos. Observaram queda de $30,3 \%$ e $2,0 \%$, respectivamente, no $\mathrm{VEF}_{1}$ pós-exercício.

A patogênese da asma induzida pelo exercício está relacionada com os fluxos de calor e água que ocorrem dentro da árvore traqueobrônquica durante ventilação com grandes volumes de ar. Embora haja controvérsias, o grosso das evidências recentes indica que ocorre queda na temperatura das vias aéreas durante hiperpnéia, seguida de imediato reaquecimento quando a ventilação diminui. Alta ventilação, particularmente em combinação com a baixa temperatura do ar inspirado e, portanto, com o baixo conteúdo de água, desloca o processo de condicionamento do ar inspirado das vias aéreas superiores para as inferiores. Não importa o que leva ao aumento da ventilação. Tanto o exercício como a hiperventilação voluntária, com manutenção do nível de anidrido carbônico, produzem o mesmo perfil térmico intratorácico e limitação do fluxo aéreo quando as variáveis apropriadas são uniformizadas ${ }^{(11)}$.

Coincidentemente, o nosso grupo com AIE, à custa de aumento proporcionalmente maior do volume corrente, atingiu nivel de ventilação significativamente maior que o grupo de asmáticos sem BIE. Observou-se também ótima correlação $(r=0,8989, p=0,015)$ nesse grupo entre o percentual de queda do $\mathrm{VEF}_{1}$ pós-exercício e a ventilação minuto máxima (Gráfico 2), diferentemente do grupo II.
Assim, na população estudada, quanto maior a ventilação atingida, maior foi a probabilidade de ser desenvolvida obstrução no fluxo aéreo. Noviski et al. ${ }^{(21)}$ observaram que a intensidade do exercício, traduzida pelo $\dot{\mathrm{V}}_{2}$, desempenha o papel principal na determinação da gravidade da AIE e que as condições climáticas agem como fatores modificadores. No entanto, no presente estudo, a correlação entre o delta $\mathrm{VEF}_{1}$ e o $\dot{\mathrm{V}}_{2} \max$ foi de $\mathrm{r}=0,3538$ $(p=0,49)$ e $r=0,4047$ ( $p=0,32)$ nos grupos I e II, respectivamente. Os nossos dados confirmam os estudos de Thio et al. ${ }^{(22)}$, que não encontraram relação entre a queda do $\mathrm{VEF}_{1}$ e o $\mathrm{VO}_{2} \max$, concluindo que a aptidão cardiovascular normal não evita a AIE.

Em asmáticos, as respostas cardiorrespiratórias ao esforço podem ser semelhantes às dos indivíduos normais ${ }^{(23)}$. $\mathrm{O} \dot{\mathrm{V}}_{2} \max (\mathrm{mL} / \mathrm{kg} / \mathrm{min})$ dos pacientes que constituíram o grupo I correspondeu a $93,5 \%$ do valor predito, enquanto jovens normais, com idade média de 16 anos, estudados em nosso laboratório com o mesmo protocolo, atingiram $\dot{\mathrm{vO}}_{2} \max$ de $91,7 \%{ }^{(14)}$. Mas essas respostas podem estar alteradas nos pacientes sedentários e/ou que temem que o esforço físico possa exacerbar sua doença. Os asmáticos menos condicionados podem melhorar sua aptidão física por meio de um programa de treinamento físico. Cochrane e Clark ${ }^{(24)}$ observaram aumento no $\mathrm{vO}_{2} \max$ de $62 \%$ para $76 \%(p=0,001)$ em 18 asmáticos submetidos a um programa de treinamento físico com duração de três meses. Outros pesquisadores observaram igualmente melhora na capacidade aeróbia com o treinamento físico ${ }^{(25-27)}$. Entretanto, Bundgaard et al. ${ }^{(25)}$ e Neder et $a l .{ }^{(27)}$ não verificaram redução na ocorrência de BIE após o período de treinamento físico.

Em suma, os nossos resultados e os dados da literatu$\mathrm{ra}^{(22,28)}$ mostraram que uma parcela dos asmáticos tem aptidão cardiorrespiratória normal durante o exercício progressivo máximo. Entretanto, na população por nós estudada, a aptidão física normal não evitou o BIE e, em virtude dos maiores volumes ventilatórios alcançados, deve ter sido fator contribuinte para o desenvolvimento da AIE.

\section{REFERÊNCIAS}

1. Anderson SD. Asthma provoked by exercise, hyperventilation and the inhalation of non-isotonic aerosols. In: Barnes PJ, Rodger IW, Thomson NC, eds. Asthma: basic mechanisms and clinical management. $3^{\text {rd }}$ ed. London: Academic Press, 1998;569-587.

2. Anderson SD, Silverman M, Konig P, Godfrey S. Exercise-induced asthma - A review. Br J Dis Chest 1975;69:1-39.

3. McFadden Jr ER. Exercise performance in the asthmatic. Am Rev Respir Dis 1984;129(Suppl):S84-S87.

4. Reiff DB, Choudry NB, Pride NB, Ind PW. The effect of prolonged submaximal warmup exercise on exercise-induced asthma. Am Rev Respir Dis 1989;139:479-484.

5. McFadden Jr ER, Ingram Jr RH. Exercise-induced asthma - Observations on the initiating stimulus. N Engl J Med 1979;301:763-769.

6. Eggleston PA, Rosenthal RR, Anderson AS, et al. Guidelines for the methodology of exercise challenge testing of asthmatics. J Allergy Clin Immunol 1979;64(pt 2):642-645.

7. Cropp GJA. The exercise bronchoprovocation test: standardization of procedures and evaluation of response. J Allergy Clin Immunol 1979; 64(pt 2):627-633.

8. Godfrey S, Konig P. Exercise-induced bronchial lability in atopic children and their families. Ann Allergy 1974;33:199-205.

9. Bierman CW, Kawabori I, Pierson WE. Incidence of exercise-induced asthma in children. Pediatrics 1975;56(Suppl):847-850.

10. Godfrey S. Exercise-induced asthma - Clinical, physiological, and therapeutic implications. J Allergy Clin Immunol 1975;56:1-17. 
11. McFadden ER Jr, Gilbert IA. Exercise-induced asthma. N Engl J Med 1994;330:1362-1367.

12. American Thoracic Society: Standards for the diagnosis and care of patients with chronic obstructive pulmonary disease (COPD) and asthma. Am Rev Respir Dis 1987;136:225-243.

13. National Heart, Lung, and Blood Institute, National Institutes of Health Guidelines for the Diagnosis and Management of Asthma. Expert Panel Report 2. Publication 1997;97-4051.

14. Valença LM, Souza AF, Rodrigues OAS, Lamar F RA, Fontana KE. Avaliação da aptidão física em atletas com teste cicloergométrico progressivo. J Pneumol 1991;17:7-12.

15. Morris JF, Koski A, Johnson LC. Spirometric standards for healthy nonsmoking adults. Am Rev Respir Dis 1971;103:57-67.

16. Jones NL, Campbell EJM. Clinical exercise testing. $2^{\text {nd }}$ ed. Philadelphia: WB Saunders, 1981

17. Jones NL, Makrides L, Hitchcock C, Chypchar T, McCartney. Normal standards for an incremental progressive cycle ergometer test. Am Rev Respir Dis $1985 ; 131: 700-708$.

18. Buckley JM, Souhrada JF, Kopetzky MT. Detection of airway obstruction in exercise-induced asthma. Chest 1974;66:244-251.

19. Lloyd TC Jr, Wright GW. Evaluation of methods used in detecting changes of airway resistance in man. Am Rev Respir Dis 1963;87:529-537.

20. Tal A, Pasterkamp H, Chernick,V. Endogenous opiates and response to exercise in asthmatic children and adolescents. Pediatr Pulmonol $1985 ; 1: 46-51$
21. Noviski N, Bar-Yishay E, Gur I, Godfrey S. Exercise intensity determines and climatic conditions modify the severity of exercise-induced asthma. Am Rev Respir Dis 1987;136:592-594.

22. Thio BJ, Nagelkerke AF, Ketel AG, Keeken BL, Dankert-Roelse, JE. Exercise-induced asthma and cardiovascular fitness in asthmatic children. Thorax 1996;51:207-209.

23. Ienna TM, McKenzie DC. The asthmatic athlete: metabolic and ventilatory responses to exercise with and without pre-exercise medication. Int J Sports Med 1997;18:142-148.

24. Cochrane LM, Clark CJ. Benefits and problems of a physical training program for asthmatic patients. Thorax 1990;45:345-351.

25. Bundgaard A, Ingemann-Hansen T, Schmidt A, Halkjaer-Kristensen J. Effect of physical training on peak oxygen consumption rate and exercise-induced asthma in adult asthmatics. Scand J Clin Lab Invest 1982; 42:9-13

26. Emtner M, Herala M, Stalenheim G. High intensity physical training in adults with asthma. A 10-week rehabilitation program. Chest 1996; 109:323-330.

27. Neder JA, Nery LE, Silva AC, Cabral ALB, Fernandes ALG. Short term effects of aerobic training in the clinical management of moderate to severe asthma in children. Thorax 1999;54:202-206.

28. Neder JA, Fernandes ALG, Silva AL, Cabral ALB, Nery LE. Relationship between aerobic fitness and clinical indicators of asthma severity in children. J Pneumol 1988;24:3-10. 